\title{
An approach for image Contrast and Resolution Enhancement based Wavelet Transform by incorporating Cuckoo Search and SVD algorithms
}

\author{
Sandeepa K S ${ }^{1}$, Basavaraj N Jagadale ${ }^{2}$, J S Bhat ${ }^{3}$ \\ ${ }^{1}$ lecturer Department of Electronics Kuvempu University Shimoga \\ ${ }^{2}$ Assistant professor Department of Electronics Kuvempu university Shimoga \\ ${ }^{3}$ The Director IIIT surat
}

\begin{abstract}
:
Image enhancement techniques are prominently used to analyze the image by enhancing key factors like contrast, resolution, and quality of the image. The proper analysis of images, it is desirable to pre-process the image for resolution and contrast enhancement. We present here a new approach based on discrete wavelet transform (DWT), singular value decomposition (SVD) for image contrast and resolution enhancement, The contrast of the image is enhanced by maximum value fusion technique applied to the images created by using modified cuckoo search algorithm (CSA) and singular value decomposition separately. The masking approach is employed, for obtaining residual pixel value between original and scaled images independently. Resolution of the image is enhanced by combining interpolated highfrequency sub-band and maximum value fusion image. The proposed algorithm helps to minimize the noise artifacts and over enhancement problems. Experimental results are tested in terms of peak signal to noise ratio (PSNR) and absolute mean brightness error (AMBE). The proposed method shows better performance compared to other contrast and resolution enhancement techniques.
\end{abstract}

Keywords: DWT; Cuckoo search algorithm; SVD; Masking; Fusion; IDW.

\section{Introduction}

An image enhancement is a process of improving the image quality without knowing its source of degradation. The image enhancement technique has been used many applications such as satellite image processing, geographic image processing, and medical image processing. The contrast and resolution are the key issues in an enhancing a digital image, in which the visual system is more sensitive to contrast, so contrast enhancement is more important in addition to resolution. The contrast enhancement helps to improve the clarity of the image. Contrast enhancement is considered as intensity based enhancements. Intensity enhancement has been realized through techniques based on the histogram, transform domain and masking approaches. The Histogram equalization (HE) is simple and effective approaches, it generates uniform histogram [1] but later on, the discrete wavelet transform and singular value decomposition based methods are proposed for contrast [2] and resolution enhancement [3].

The DCT-SVD and DWT-SVD based illumination enhancement presented by updating singular value matrix of singular value decomposition (SVD). In these methods, low-low (LL) sub-band of an input image is considered by applying DWT [4, 5]. Recently algorithms are proposed on masking based contrast enhancement [6], has internal scaling processes, whereas Un-Sharpe masking technique has fixed scale value for masking process [7].

In this paper, we tried to improve upon our previous work [8], here we have addressed both contrast and resolution enhancement by scaling the reconstructed approximation coefficients using cuckoo search algorithm and SVD approach to achieve better results. This is a double masking approach for image 
enhancement by preserving minute details of the image and sensitive edges. These methods shows superior result than conventional and state-of-art scheme of contrast enhancement technique (i.e. bi-histogram equalization (BBHE) and dualistic sub image histogram equalization (DSIHE), exposure-based sub image histogram equalization (ESIHE), non-parametric modified histogram equalisation (NMHE), singular value decomposition (SVD), standard intensity deviation clipped sub image histogram equalization (SIDCSIHE), and contrast enhancement by scaling reconstruction approximation coefficient using SVD (CE_SRA_SVD)) [9-14]. The proposed method outperform over conventional and state-of-art technique of resolution enhancement technique (i.e. the nearest neighbour, bilinear, bi-cubic, wavelet zero padding (WZP), Regularity-preserving image interpolation, new edge-directed interpolation (NEDI), hidden Markov model (HMM), HMM-based image super-resolution (HMM SR), WZP and cycle-spinning (WZP-CS), DWT based super-resolution (DWT SR), complex wavelet transform based super-resolution (CWT SR), and modified context-based image interpolation (MCBII)) [15-22]. The paper is structured as follows, section 2 contains the proposed method and section 3 we discuss experimental results. The conclusions are given in section 4 .

\section{The Proposed Methodology}

This paper presents an improved contrast and resolution enhancement method by using DWT, CSR, SVD, masking approach, and maximum value fusion method. In this work contrast and resolution, enhancement is carried out mainly in three steps. In the first step, the low contrast and resolution image undergoes DWT decomposition and produce four sub-band namely LL, LH, HL, and HH. In which, the illumination information is enclosed in LL sub-band and other sub-bands (LH, HL, and HH) contain edge information. The contrast enhancement is achieved by manipulating LL sub-band using CSR, SVD, masking approach. The two individual contrast-enhanced images are obtained by scaling the reconstruction approximation coefficients of DWT using CSR algorithm and SVD method followed by masking approach. In the second step, the best intensity level is selected by applying maximum fusion approach. The last one is resolution enhancement is carried out by applying bi-cubic interpolation method to all the sub-band and the resulting subbands are subjected to IDWT to produce a high-resolution output image. The block diagram of the proposed method is shown in figure 1 and the process is explained in detail. In this proposed algorithm, the contrast and resolution of the image are enhanced by minimizing the loss through preserving edge information which affects the quality of the enhanced image.

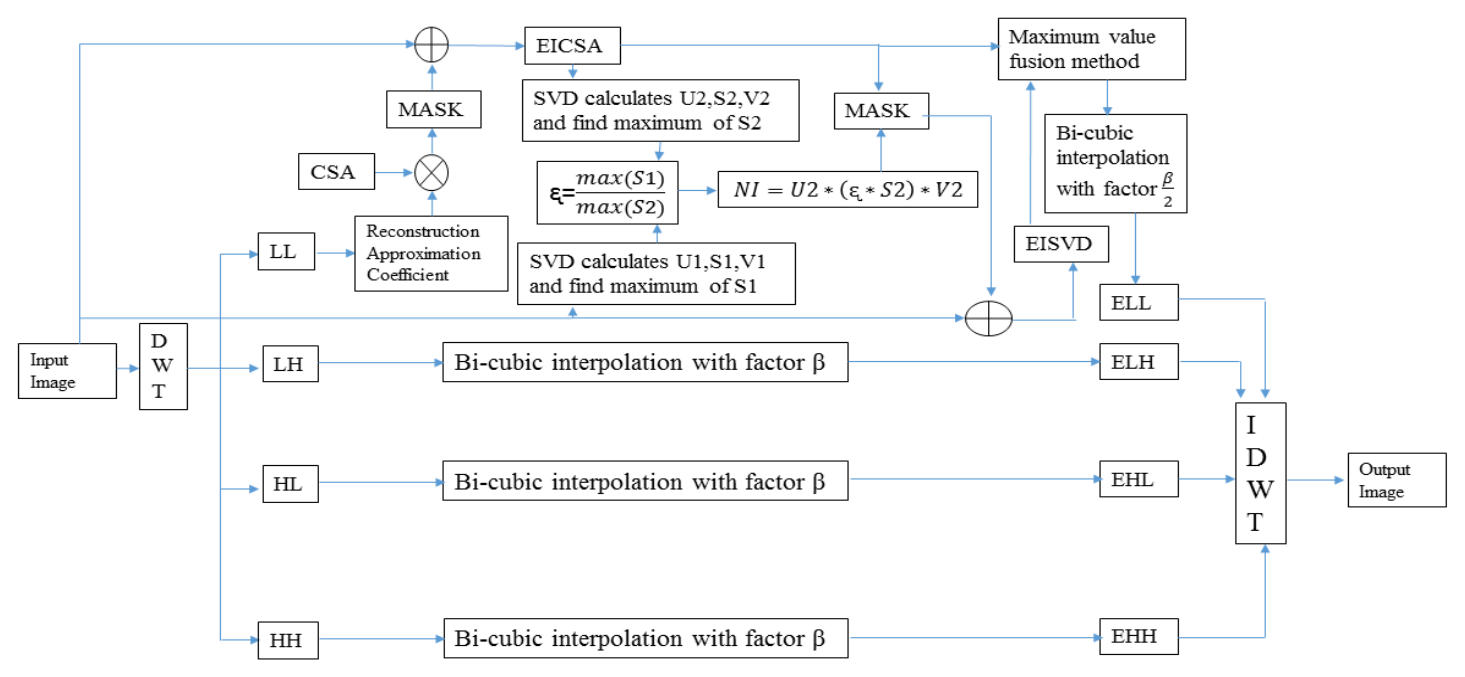

Figure 1. Block diagram of the proposed method.

Step 1. In the very first step, low contrast and resolution image have taken for processing. 
Step 2. Perform 1 level DWT to decompose the image into four subbands are LL, LH, HL, and HH. In which LL sub-band is called an approximation coefficient and remaining sub-bands are detailed coefficients. Step 3. Perform reconstruction of the approximation coefficients by using IDWT.

Step 4. The reconstruction approximation is scaled by using optimum scale value obtained by using modified cuckoo search algorithm.

Step 5. The masking formulation is obtained by subtracting the scaled image and original input image [mask].

Step 6. The Enhanced image using modified cuckoo search algorithm (EICSA) is obtained by adding mask image with original image.

Step 7. SVD is applied to EICSA image and reconstruction approximation for getting $U 1 \times S 1 \times V 1$ and $U 2 \times S 2 \times V 2$ then $\max (S 1)$ and $\max (S 2)$ obtained.

Step 8. Calculate weighting value $\varepsilon$ by using an equation

$$
\varepsilon=\frac{\max (S 1)}{\max (S 2)}
$$

Step 9. The new image (NI) is obtained by inverse singular value decomposition $N I=U 2 \times(\varepsilon \times S 2) \times V 2$. Step 10. The masking formulation is obtained by subtracting the new image and original input image [mask].

Step 11. The Enhanced image using singular value decomposition (EISVD) is obtained by adding mask image with original image.

Step 12. Maximum value fusion method is applied to select pixel value with the maximum intensity between EICSA and EISVD to generate the final image with better contrast.

Step 13. The high-frequency subbands ( $\mathrm{LH}, \mathrm{HL}$, and $\mathrm{HH})$ are interpolated using bi-cubic interpolation method the factor $\beta$ and generates estimated high-frequency sub-bands (ELH, EHL, and EHH). The maximum value fusion process is interpolated with factor $\beta / 2$ and generates estimated low-frequency subband (ELL).

Step 14. Apply IDWT using estimated sub-bands (ELL, ELH, EHL, and EHH).

Step 15. Contrast and resolution enhanced output image is obtained.

\subsection{Double masking approach based modified cuckoo search algorithm and SVD}

In the double masking approach, the masking formulation is obtained by subtracting scaled image and original image twice. At first, the reconstruction approximation coefficients scaled by using modified cuckoo search algorithm and generates EICSA image. The EISVD image obtained by applying SVD method between EICSA and reconstruction approximation coefficient.

\subsubsection{Enhanced Image using Modified Cuckoo Search Algorithm (EICSA)}

In this step, we modified the cuckoo search algorithm used in [23] to get optimized scale value. Here, we have used PSNR based fitness function to get optimized scale value and is used to scale the image recursively. The mask is derived using a scaled image and reconstructed approximation coefficients. The mask is added to the original image and generates the resultant image EICSA as shown in figure 1.

Cuckoo Search (CS) algorithm is meta-heuristic optimization algorithm based on captivating reproduction policy of the bird cuckoo developed by Yang and Deb [24]. Cuckoo lays their eggs in the host bird nest, it doesn't construct the nest. If host bird identifies cuckoo egg, it will either rebuild its nest or abandon its eggs.

The CS is based on three idealized rules:

- At a time, each cuckoo lays one egg in a randomly chosen nest.

- Next generation will carry over to best nests with a high quality of eggs (solutions).

- The number of available host nests is fixed and a host can discover an alien egg with probability 'pa' $\in[0,1]$.

The host birds either abandon the nest or throw the egg away. This implementation is carried by replacing 'pa'. Each egg corresponds to a solution and its fitness is calculated. A new solution generated using Levy flight as given in the equation 1.

$$
x_{i}(t+1)=x_{i}(t)+\alpha \otimes \operatorname{levy}(\lambda),
$$


where $\alpha$ is step size and product represents entry wise multiplication. It essentially provides a random walk while random step length is drawn from a Levy distribution as given below:

$$
\operatorname{levy}(\lambda)=t^{-\lambda}: 1<\lambda \leq 3,
$$

The iterative process continues to the replacement of a not good egg and finding an optimum solution [25], where $\mathrm{k}$ is the best nest. The flow chart of the cuckoo search algorithm is shown in figure 2.

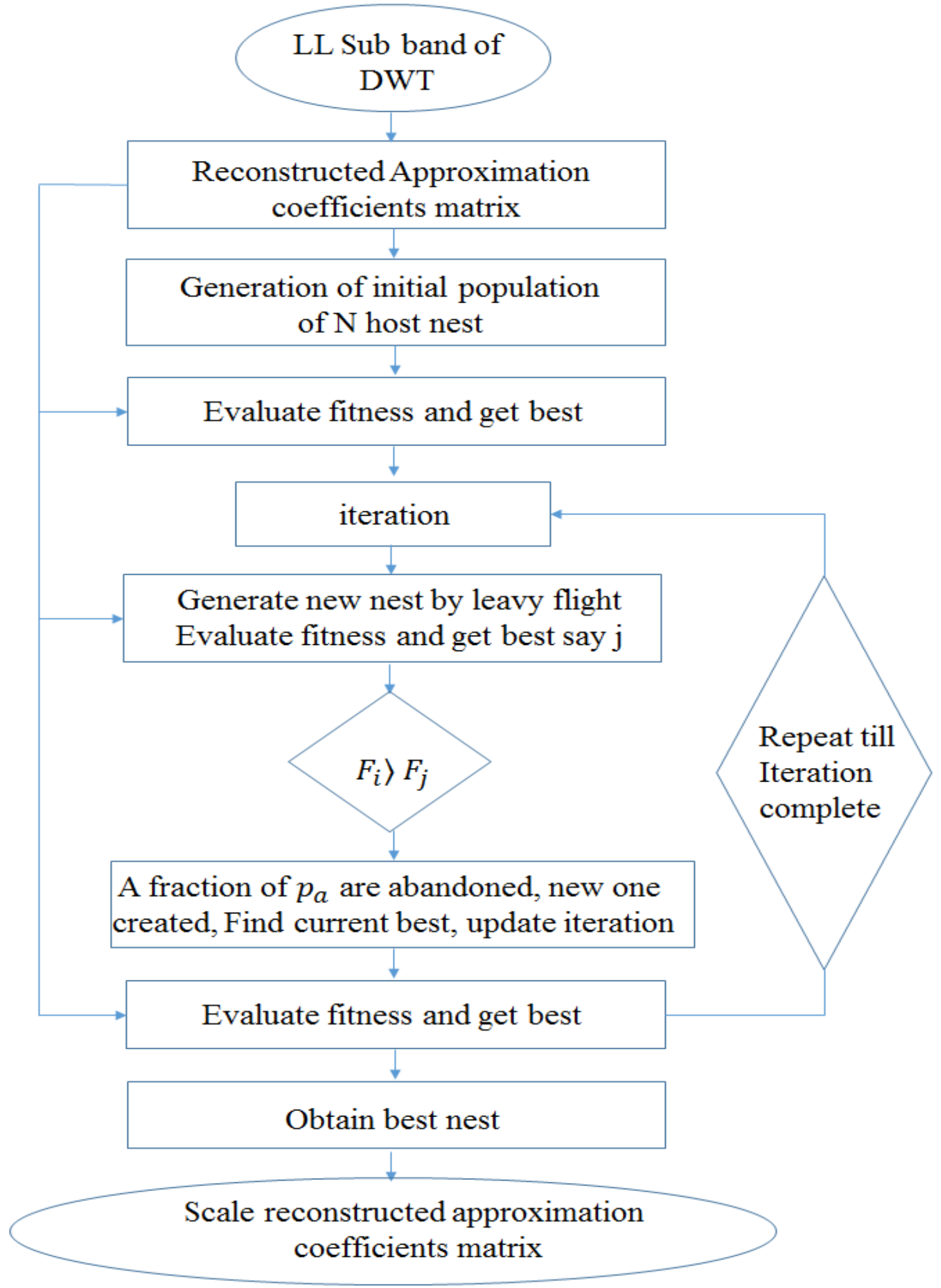

Figure 2. Block diagram of Modified cuckoo search algorithm/ method. 


\subsubsection{Enhanced Image using SVD (EISVD)}

In this approach, SVD based masking formulation is obtained by subtracting new image (NI) from EIMCSA image. This mask image is added to the original image, which generates enhanced image EISVD as shown in figure 1.

To get new image (NI), the original low resolution (LR) image and EICSA image are decomposed by SVD as given by equations (3) and (4).

$$
\begin{aligned}
& L R \text { image }=U 1 \times S 1 \times V 1 \\
& E I C S A \text { image }=U 2 \times S 2 \times V 2 .
\end{aligned}
$$

Here U1, V1, U2, and V2 are the orthogonal matrices, identified as left and right singular vector of both EICSA and LR image. S1 and S2 are diagonal matrices containing intensity information. To enhance the image, $\varepsilon_{6}$ is calculated by LR and EICSA images as given in equation (5).

$$
\varepsilon=\frac{\max (S 1)}{\max (S 2)}
$$

The $\varepsilon$ is considered as weighted value, which is highest singular value and is used to enhance the contrast of the LR image. The new image (NI) is obtained from inverse singular value decomposition [26] as given in equation (6).

$$
N I=U 1 \times \varepsilon S 1 \times V 1
$$

The masking approach used between EIMCSA and NI, the EISVD image is obtained by adding with the original image.

\subsection{Maximum value fusion process}

The fusion is the process of creating a single image by considering the intensity levels of two images. Maximum value fusion method is the most effective as every pixel of the fused image will be pixel with maximum intensity. The method is applied used for fusing EICSA and EISVD, to generate the final image with better contrast.

\section{Experimental Results}

The pre-eminence of the proposed method is illustrated by comparing both objective and subjective assessment with well-known existing methods. As far as objective assessment is concerned, the performance of the proposed method is measured in terms of PSNR, AMBE values. The subjective assessment is concerned, figures 9-12 show the experimental results of the proposed method, and are compared with other contrast and resolution enhancement methods.

\subsection{Objective assessment}

The performance of the proposed method is measured using the equations (7)-(9). Given that, (M x N) is the size of the image, $I_{i n}$ is input image, $I_{e}$ the enhanced image, $M a x_{i n}$ the maximum possible value of the input image. The PSNR has represented a measure of the peak signal to noise error between the input and enhanced image is presented in equation (7). MSE is a mean squared error between input image $I_{\text {in }}$ and enhanced image $I_{e}$ as is shown in equation (8). The AMBE equation is expressed in equation (9).

$$
\begin{gathered}
P S N R=10 \log _{10}\left(\frac{\operatorname{Max}_{i n}^{2}}{M S E}\right) \\
M S E=\frac{1}{(M X N)} \sum_{i=1}^{M} \sum_{j=1}^{N}\left(I_{i n}(i, j)-I_{e}(i, j)\right)^{2}
\end{gathered}
$$




$$
A M B E=\left|I_{\text {in }}-I_{e}\right|
$$

Table 1 shows the parameter, which has used in the cuckoo search algorithm to get optimized scale value. The table 2 indicates the comparative analysis of resultant PSNR for four well-known test images (Lena, Elaine, Baboon, and Peppers) has used to study the effectiveness of the proposed method over conventional and state-of-art resolution enhancement techniques.

The proposed method comparison of the medical images (Brain_MRI1, Face X-Ray, MRI, Ribs-Ray) in terms of PSNR value is shown in figure 8 and AMBE values as shown in table 3. The proposed method shows better performance as compared to existing resolution and contrast enhancement techniques.

The table 4, shows the quantitative performance of the proposed method for 50 mammogram images. The objective assessment shows that proposed method generates less noise artifact and has been proved by studying PSNR values. The effective brightness preservation has proved by minimum value over other techniques.

Table 1. The parameter used in the cuckoo search algorithm

\begin{tabular}{|c|c|}
\hline Parameters & Value \\
\hline Number of Nests & 50 \\
\hline Alien egg discover rate pa & 0.25 \\
\hline Number of iteration & 50 \\
\hline Lower bound value & 0 \\
\hline Upper bound value & 1 \\
\hline Dimension & 1 \\
\hline
\end{tabular}

Table 2. PSNR values of the compared methods for resolution enhancement

\begin{tabular}{|c|c|c|c|c|}
\hline Technique/Images & Lena & Elain & Pepper & Baboon \\
\hline Bilinear & 26.34 & 25.38 & 20.51 & 25.16 \\
\hline Bicubic & 26.84 & 28.93 & 20.61 & 25.66 \\
\hline WZP (db.9/7) & 28.84 & 30.44 & 21.47 & 29.57 \\
\hline $\begin{array}{c}\text { Regularity- Preserving image } \\
\text { interpolation }\end{array}$ & 28.81 & 30.42 & 21.47 & 29.57 \\
\hline NEDI & 28.81 & 29.97 & 21.18 & 28.52 \\
\hline HMM & 28.86 & 3.46 & 21.47 & 29.58 \\
\hline HMM_SR & 28.88 & 30.51 & 21.49 & 29.6 \\
\hline WZP_CS & 29.27 & 30.78 & 21.54 & 29.87 \\
\hline WZP_CS_ER & 29.36 & 30.89 & 21.56 & 30.05 \\
\hline CWT_SR & 33.79 & 33.05 & 23.12 & 31.03 \\
\hline SWT & 32.01 & 31.25 & 22.79 & 29.46 \\
\hline DWT_SVD & 29.462 & 32.907 & 26.43 & 24.94 \\
\hline MCBIIA & 31.50 & 33.42 & 25.63 & 23.23 \\
\hline CE_SRA_SVD & 30.258 & 31.495 & 26.29 & 25.66 \\
\hline PROPOSED & 31.20 & $\mathbf{3 4 . 1 7}$ & $\mathbf{2 6 . 3 3}$ & $\mathbf{3 0 . 4 5}$ \\
\hline
\end{tabular}




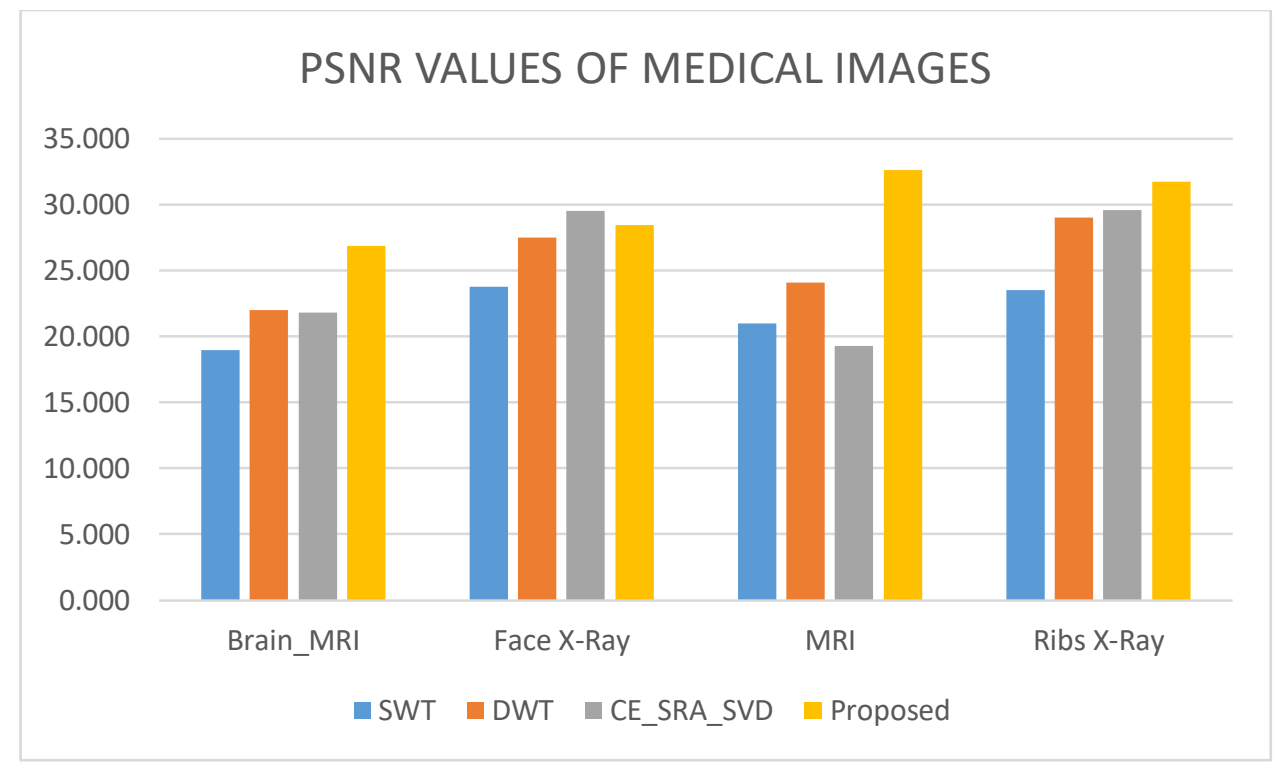

Figure 8. PSNR values of compared methods for contrast and resolution enhancement

Table 3. AMBE values of the compared methods for contrast enhancement

\begin{tabular}{|l|r|r|r|r|}
\hline Techniques/images & \multicolumn{1}{|l|}{ Brain_MRI1 } & \multicolumn{1}{|l|}{ Face X-Ray } & MRI & \multicolumn{1}{l|}{ Ribs X-Ray } \\
\hline BBHE & 29.61 & 36.22 & 27.55 & 9.94 \\
\hline DSIHE & 31.56 & 22.55 & 42.78 & 4.39 \\
\hline ESIHE & 24.02 & 24.02 & 32.57 & 2.86 \\
\hline NMHE & 21.02 & 7.56 & 36.13 & 9.17 \\
\hline SVD & 15.96 & 15.06 & 21.62 & 7.7 \\
\hline SIDCSIHE & 21.81 & 4.46 & 29.60 & 4.20 \\
\hline CE_SRA_SVD & 13.6 & 1.98 & 18.75 & 4.49 \\
\hline PROPOSED & $\mathbf{0 . 1 8 6}$ & $\mathbf{0 . 1 8 9}$ & $\mathbf{0 . 1 6 9}$ & $\mathbf{0 . 1 5 4}$ \\
\hline
\end{tabular}

Table 4. PSNR and AMBE value of 50 mammogram images

\begin{tabular}{|l|l|l|}
\hline Methods & Average PSNR & Average AMBE \\
\hline DWT_RE & 26.34 & 12.12 \\
\hline SWT & 25.58 & 11.57 \\
\hline CE_SRA_SVD & 33.27 & 03.77 \\
\hline Proposed method & $\mathbf{3 6 . 7 3}$ & $\mathbf{0 . 0 1 2}$ \\
\hline
\end{tabular}

\subsection{Subjective assessment}

The performance of the proposed method is analyzed by processing some medical images (Brain_MRI1, Face X-ray), grey scale image (Elain, pepper) and mammogram images.

Figure 9a-f are the results of the proposed method steps from the original image to enhanced image. The figure $9 \mathrm{a}$ is the low-resolution input image and figure $9 \mathrm{~b}$ is reconstructed approximation coefficients of the LL subband. Figure 9c is the mask image. Figure 9d-e are the EICSA and EISVD images and Figure 9f is the resultant, contrast-enhanced image with better visual quality than the original image and is visible in the portion of the nasal cavity, sphenoid sinus, eye, cerebellar magna, a posterior element of c4 and vertebra surrounding. The cingulate sulcus and precentral sulcus portion are enhanced. 


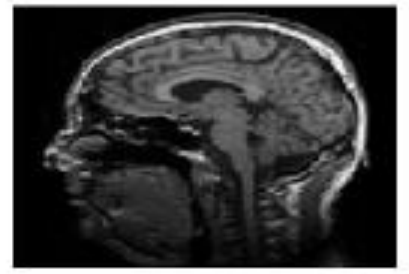

(a)

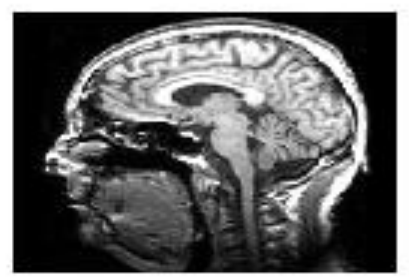

(d)

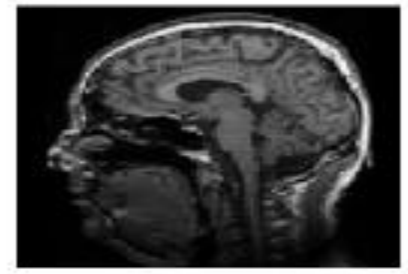

(b)

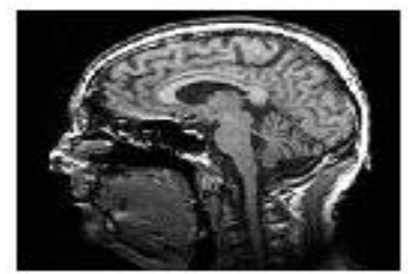

(e)

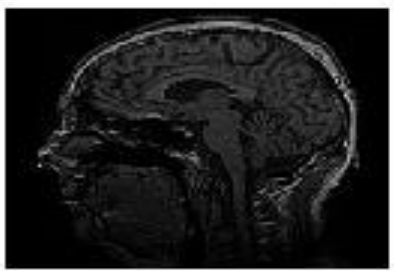

(c)

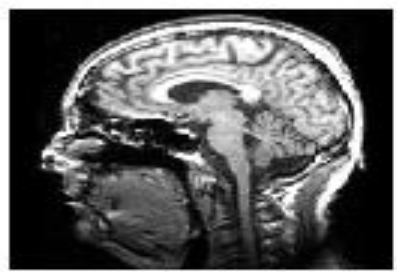

(f)

Figure 9. Results of different stages of proposed method (a) original low contrast an resolution image; (b) Reconstructed Approximation coefficient; (c) Mask image; (d) EICSA; (e) EISVD; (f) Fusion image of EICSA and EISVD.

The resolution enhanced results of Face X-Ray are shown in figure 10a-h. Figure 10b, c are processed by DWT_RE, SWT_RE individually and yield darker images. Figure 10d, e are the result of DWT_SVD and CE_SRA_SVD, have acceptable contrast enhancement. The proposed method as shown in figure 10f has improved contrast and resolution enhancement as compared to other methods.

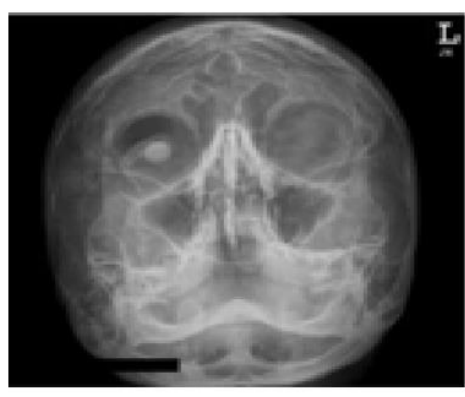

(a)

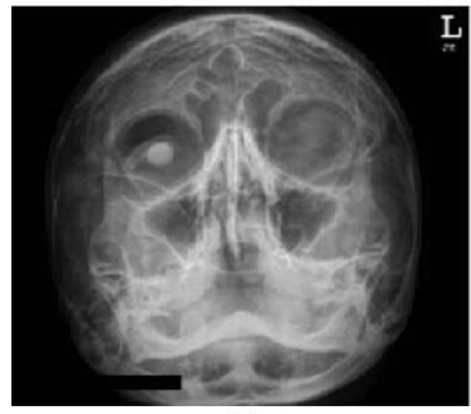

(d)

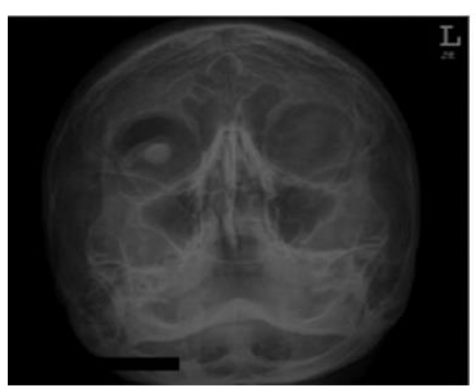

(b)

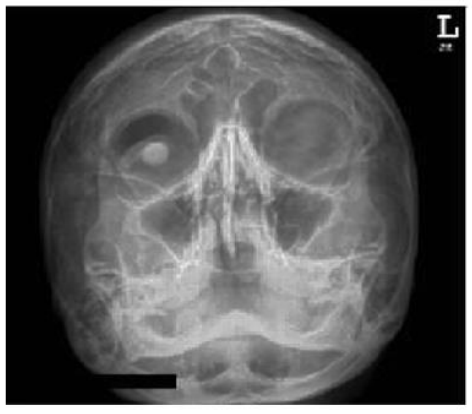

(e)

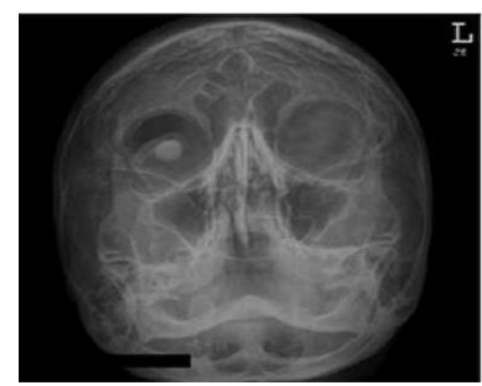

(c)

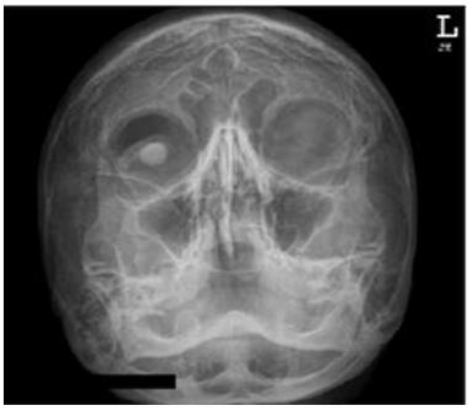

(f)

Figure 10. Results of different methods for the Face X-Ray image (a) original low contrast and resolution image (128x128); (b) DWT_RE (512x512); (c) SWT_RE (512x512) (d) DWT_SVD (512x512); (e) CE_SRA_SVD; (f) proposed method $(512 \times 12)$.

Figure 11a is the low-resolution elain image and figure $11 \mathrm{~b}, \mathrm{c}$ is the resolution enhanced results of DWT_RE and SWT_RE individually. The result of these two methods are effectively enhanced image resolution but failed to improve its contrast. The figure 11d, e, represent the methods DWT_SVD, CE_SRA_SVD, have failed to enhance the image contrast and resolution. The superiority of the proposed method as shown in figure 11f, which enhances both contrast and resolution without affecting image 
features.

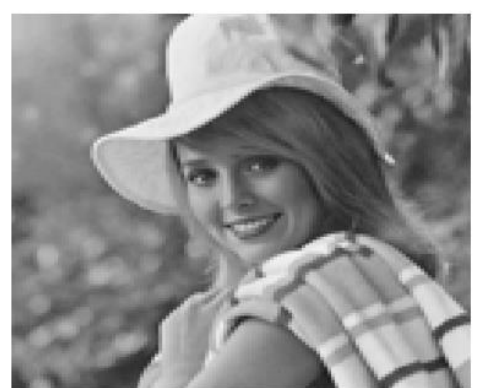

(a)

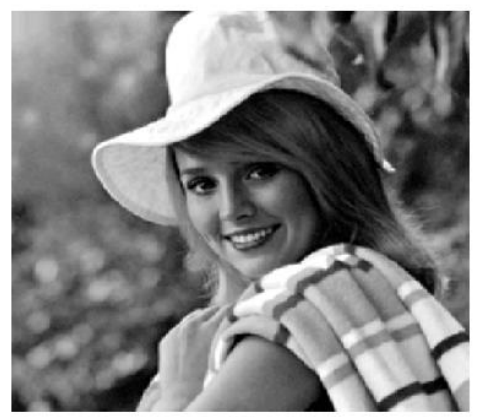

(d)

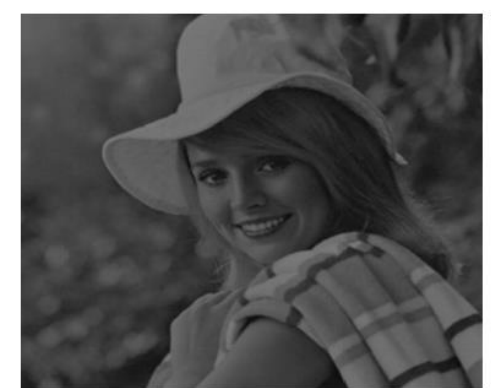

(b)

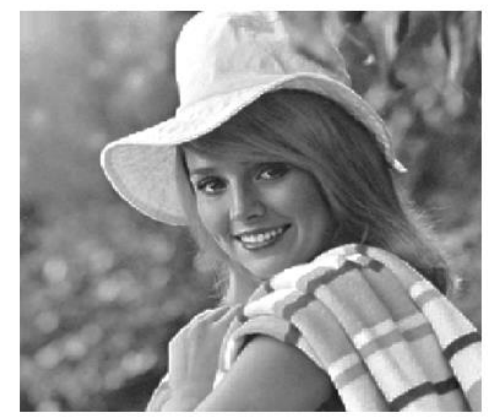

(e)

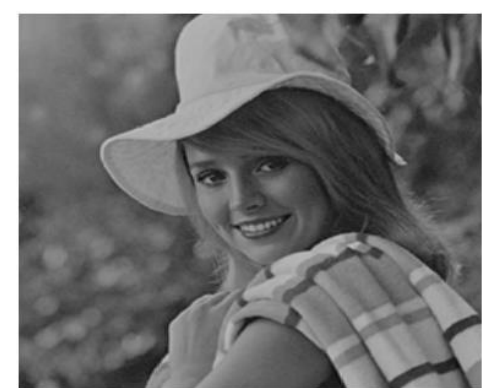

(c)

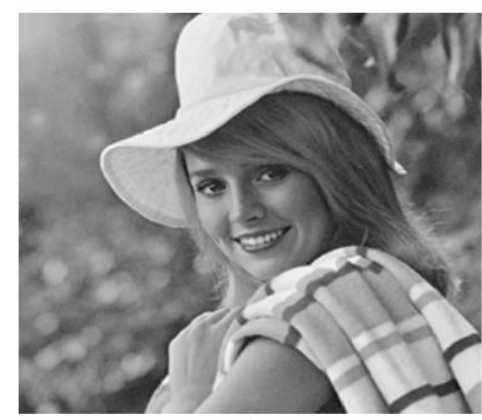

(f)

Figure 11. Results of different methods for the Elain image (a) original low contrast and resolution image (128x128); (b) DWT_RE $(512 \times 512)$; (c) SWT_RE (512x512) (d) DWT_SVD $(512 \times 512)$; (e) CE_SRA_SVD ; (f) proposed method (512x12).

The resolution enhanced results of mammogram images are shown in figure 12a-f. Figure 12b, $c$ are processed by DWT_RE, SWT_RE, individually are darker images and fail to show normal fatty tissue, dense breast tissue and infected area clearly. Figure 12d, e is the result of DWT_SVD and CE_SRA_SVD have acceptable contrast enhancement as compare to other methods. Figure $12 \mathrm{f}$ is a result of proposed methods have natural look in both contrast and resolution enhancement.

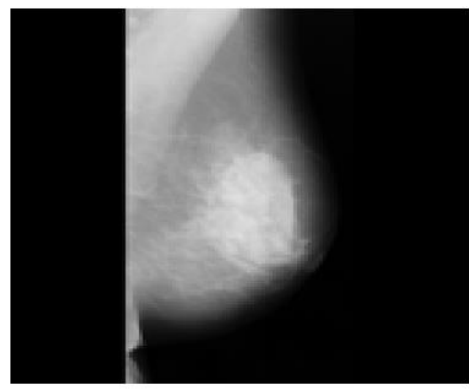

(a)

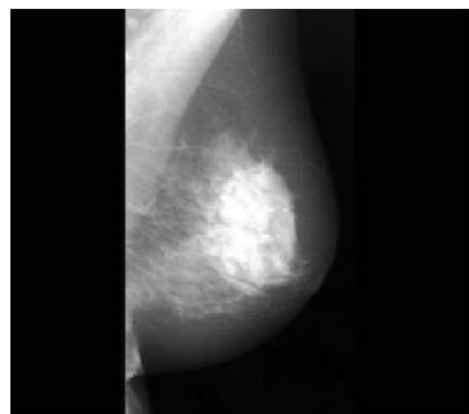

(d)

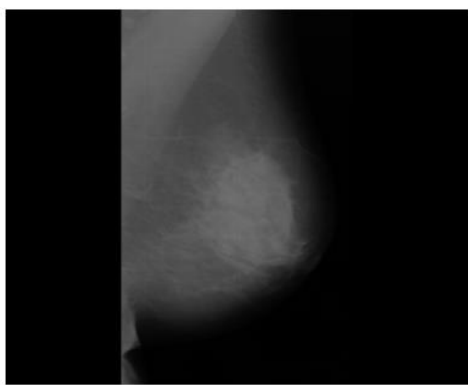

(b)

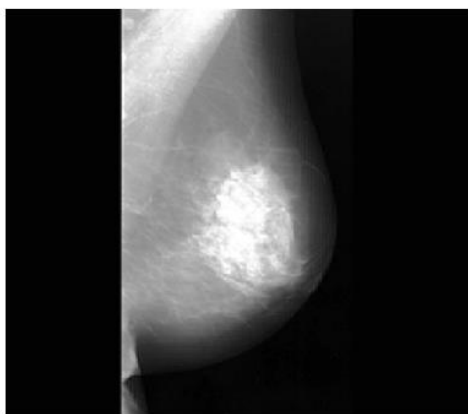

(e)

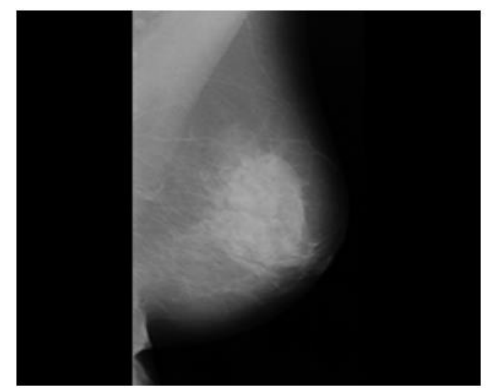

(c)

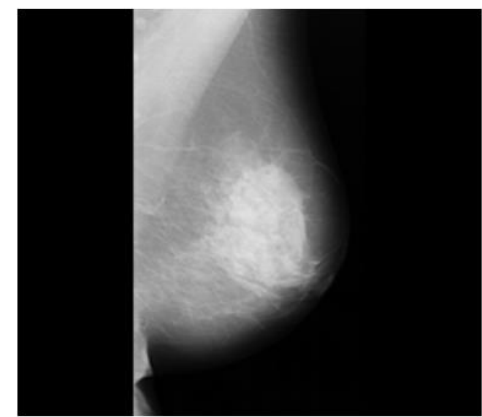

(f)

Figure 12. Results of different methods for the mammogram image (a) original low contrast and resolution image (128x128); (b) DWT_RE (512x512); (c) SWT_RE (512x512) (d) DWT_SVD (512x512); (e) CE_SRA_SVD; (f) proposed method $(512 \times 12)$. 
Figure 13 a-d, are the pepper image obtained by applying DWT_RE, DWT_SVD, and proposed method individually, figure 13 e-f are crop portion of applying DWT_RE, DWT_SVD, and proposed method individually. The results of DWT and DWT_SVD images important features are not visible and introduced blurring effect. Figure $13 \mathrm{~d}$ and $\mathrm{h}$ is the result of the proposed method which enhances the contrast and resolution. It can observe in the resultant image and on its cropping portion.

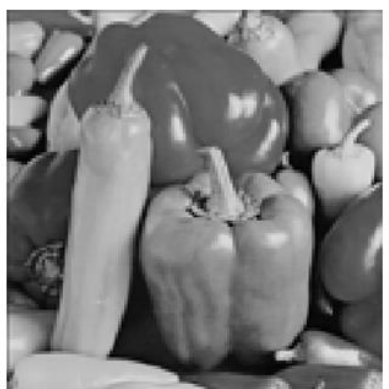

(a)

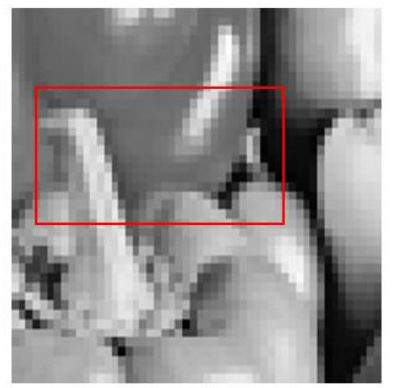

(e)

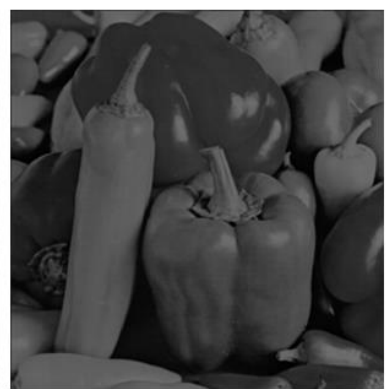

(b)

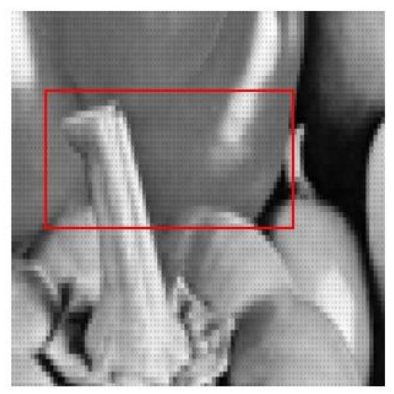

(f)

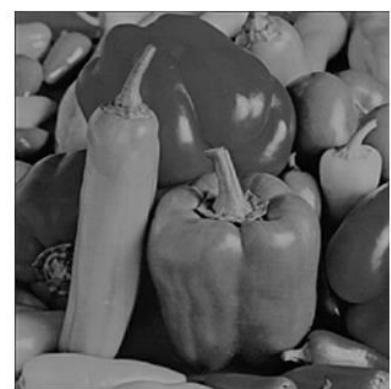

(c)

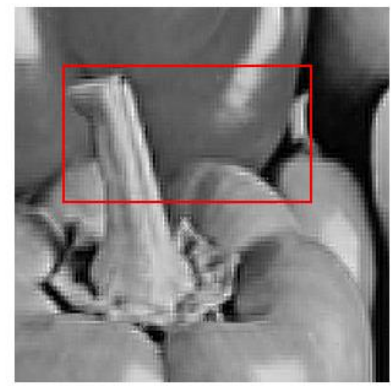

(g)

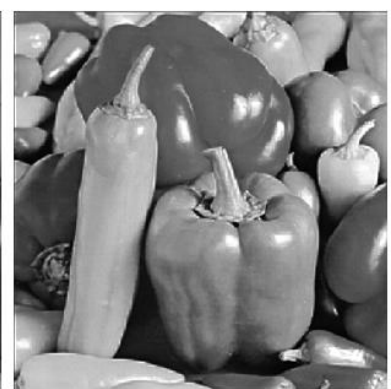

(d)

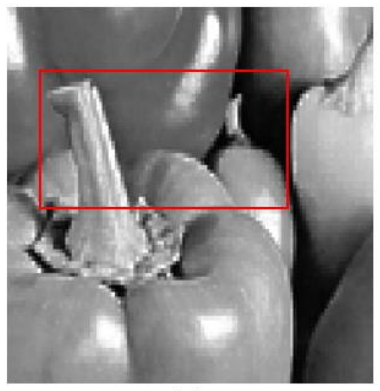

(h)

Figure 13. Results of different methods for the pepper image (a) original low contrast an resolution image; (b) DWT_RE (512x512); (c) SWT_RE (512x512) (d) proposed method (512x512); (e) original image crop portion; (f) DWT_RE crop portion;(g) DWT_SVD crop portion; (h) proposed method crop portion.

\section{Conclusions}

In this paper, we have proposed a new method, based on wavelet transform, for contrast and resolution enhancement. For contrast improvement, cuckoo search algorithm \& SVD are used. The resolution of the image is improved by applying interpolation method. The proposed algorithm helps to minimize the noise artifacts and blurring. Experimental results are tested by measuring peak signal to noise ratio (PSNR) and absolute mean brightness error (AMBE) and are found to be significantly better as compared to other methods.

Conflict of interest: The author declares no conflict of interest.

\section{References}

1. Gonzalez, R.C.; Woods, R. Digital Image Processing, 3rd ed.; Pearson, INDIA, 2014, pp. 144-166.

2. Demirel, H.; Anbarjafari, G.; Jahromi,M.N.S.Image Equalization based on singular value decomposition. IEEE international symposium on computer and information sciences Oct 2008, 27-29, pp.1-5.

3. Demirel, H.; Anbarjafari, G. Satellite image resolution enhancement using complex wavelet transform. IEEE Geoscience and Remote Sensing Letter Jan 2010, 7, 123-126.

4. Bhandari A K, Gadde M, Kumar A, Singh, GK. Comparative analysis of different wavelet filters for low contrast and brightness enhancement of multispectral remote sensing images. In: Proceedings of the IEEE international conference on machine vision and image processing (MVIP), p.81-6; 2012.

5. Bhandari, A.K.; Kumar, A.; Padhy, P.K. Enhancement of low contrast satellite images using discrete cosine transform and singular value decomposition. World AcadSciEng Technology 2011; 79:35-41. 
6. Daniel, E.; Anitha, J. Optimum wavelet-based masking for contrast enhancement of medical images using enhanced cuckoo search algorithm.Elsevier, computers in biology and medicine 2016, 71, 419155.

7. Polesel, A.; Ramponi,g.; Mathews, V.J. Image enhancement via adaptive unsharp masking. ", IEEE Transactions on Image Processing Mar 2000, 9, issue 3.

8. Sandeepa K S, Basavaraj N Jagadale, J S Bhat. Wavelet-based medical image contrast and resolution enhancement by incorporating cuckoo search algorithm and SVD. 5th IIAE International Conference on Intelligent Systems and Image Processing 2017, DOI: 10.12792/icisip2017.034.

9. C.Zuo, C.; Chen, O.; sui,X.Range limited bi-histogram equalization for image contrast enhancement. Optic 2013, 124, 425-431.

10. Yu Wang.; Quinchen, BaeominZang. Image enhancement based on equal area dualistic sub-image histogram equalization method. IEEE Transactions on Consumer Electronics Feb 1999, 45, issue 1.

11. Kuldeep, singh. Rajiv, Kapoor. Image Enhancement using Exposure Based Sub Image Histogram Equalization. Pattern Recognition Letter 2014, 36, 10-14.

12. Nmhe

13. Sandeepa K S, Basavaraj N Jagadale, and J S Bhat, "Standard Intensity Deviation Approach based Clipped Sub Image Histogram Equalization Algorithm for Image Enhancement" International Journal of $\begin{array}{lllll}\text { Advanced } & \text { Computer } & \text { Science and }\end{array}$ http://dx.doi.org/10.14569/IJACSA.2018.090116

14. Sandeepa K S, Basavaraj N Jagadale, J S Bhat, Mukund N Naragund and Panchaxri, "Image Contrast Enhancement by Scaling Reconstructed Approximation Coefficients using SVD Combined Masking Technique" International Journal of Advanced Computer Science and Applications(IJACSA), 9(2), 2018. http://dx.doi.org/10.14569/IJACSA.2018.090218

15. W. K. Carey, D. B. Chuang, and S. S. Hemami, "Regularity-preserving image interpolation," IEEE Trans. Image Process., vol. 8, no.9, pp. 1295-1297, Sep. 1999.

16. X. Li and M. T. Orchard, "New edge-directed interpolation," IEEE Trans. Image Process., vol. 10, no. 10, pp. 1521-1527, Oct. 2001.

17. K. Kinebuchi, D. D. Muresan, and R. G. Baraniuk, "Wavelet-based statistical signal processing using hidden Markov models," in Proc. Int. Conf. Acoust., Speech, Signal Process., 2001, vol. 3, pp. 7-11.

18. S. Zhao, H. Han, and S. Peng, "Wavelet domain HMT-based image super-resolution," in Proc. IEEE Int. Conf. Image Process., Sep. 2003, vol. 2, pp. 933-936.

19. A. Temizel and T. Vlachos, "Wavelet domain image resolution enhancement using cycle-spinning," Electron. Letter. vol. 41, no. 3, pp. 119-121, Feb. 3, 2005.

20. A. Temizel and T. Vlachos, "Image resolution upscaling in the wavelet domain using directional cycle spinning," J. Electron. Image. vol. 14, no. 4, 2005.

21. A .F. D. Araujo, et al., New artificial life model for image enhancement, Expert Syst. Appl.41 (2014)5892-5906.

22. Sandeepa K S., B N Jagadale and J S Bhat. A Modified Context based Image Interpolation Algorithm for Digital Images. International Journal of Computer Applications 171(2):34-37, August 2017.

23. Bhandaria, A.k; Sonia, V.; H.; Kumaran, A.; Singhb, G.K.Cuckoo search algorithm based satellite image contrast and brightness enhancement using DWT-SVD. ISA Transactions 2014, 53, 1286-1296.

24. Yang, X.S.; Deb, S. Cuckoo Search via levy flights in proceedings of the world congress on nature and biological inspired computing (NaBIC). IEEE publications, 2009,pp,210-214.

25. Shilpha Suresh, Dr.Shyam Lal, "An Efficient Cuckoo Search Algorithm based Multilevel Thresholding for Segmentation of Satellite Images Using Different Objective Functions", Expert Systems with Applications $58 \cdot$ April 2016,184-209.

26. Iqbal, M.Z.; Ghafoor, A.; Siddiqui, A.M; Riaz, M.M.; Khalid, U.Dual-tree complex wavelet transform and SVD based medical image resolution enhancement. Signal Processing Dec 2014, 105, Pages 430 437. 